\title{
Modulation of monocarboxylate transporter 8 oligomerization by specific pathogenic mutations
}

\author{
Jana Fischer*, Gunnar Kleinau*, Anne Müller, Peter Kühnen, Denise Zwanziger², \\ Anita Kinne', Maren Rehders ${ }^{3}$, Lars C Moeller ${ }^{2}$, Dagmar Führer ${ }^{2}$, Annette Grüters, \\ Heiko Krude, Klaudia Brix ${ }^{3}$ and Heike Biebermann
}

Institut für Experimentelle Pädiatrische Endokrinologie, Charité-Universitätsmedizin Berlin, Augustenburger Platz 1, 13353 Berlin, Germany

${ }^{1}$ Leibniz-Institut für Molekulare Pharmakologie, Berlin, Germany

${ }^{2}$ Universitätsklinikum Essen, Klinik für Endokrinologie und Stoffwechselerkrankungen, Essen, Germany

${ }^{3}$ Jacobs University Bremen, Bremen, Germany

*(J Fischer and G Kleinau contributed equally to this work)

Correspondence should be addressed to H Biebermann Email Heike.Biebermann@ charite.de

\begin{abstract}
The monocarboxylate transporter 8 (MCT8) is a member of the major facilitator superfamily (MFS). These membrane-spanning proteins facilitate translocation of a variety of substrates, MCT8 specifically transports iodothyronines. Mutations in MCT8 are the underlying cause of severe X-linked psychomotor retardation. At the molecular level, such mutations led to deficiencies in substrate translocation due to reduced cell-surface expression, impaired substrate binding, or decreased substrate translocation capabilities. However, the causal relationships between genotypes, molecular features of mutated MCT8, and patient characteristics have not yet been comprehensively deciphered. We investigated the relationship between pathogenic mutants of MCT8 and their capacity to form dimers (presumably oligomeric structures) as a potential regulatory parameter of the transport function of MCT8. Fourteen pathogenic variants of MCT8 were investigated in vitro with respect to their capacity to form oligomers. Particular mutations close to the substrate translocation channel (S194F, A224T, L434W, and R445C) were found to inhibit dimerization of MCT8. This finding is in contrast to those for other transporters or transmembrane proteins, in which substitutions predominantly at the outer-surface inhibit oligomerization. Moreover, specific mutations of MCT8 located in transmembrane helix 2 (del230F, V235M, and ins236V) increased the capacity of MCT8 variants to dimerize. We analyzed the localization of MCT8 dimers in a cellular context, demonstrating differences in MCT8 dimer formation and distribution. In summary, our results add a new link between the functions (substrate transport) and protein organization (dimerization) of MCT8, and might be of relevance for other members of the MFS. Finally, the findings are discussed in relationship to functional data combined with structural-mechanistical insights into МСТ8.
\end{abstract}

\section{Key Words}

monocarboxylate transporter 8

- oligomerization

- pathogenic mutations

- major facilitator superfamily http://jme.endocrinology-journals.org DOI: 10.1530/JME-14-0272
๑ 2015 Society for Endocrinology Printed in Great Britain
Published by Bioscientifica Ltd.
Journal of Molecular

Endocrinology

(2015) 54, 39-50 


\section{Introduction}

Members of the major facilitator superfamily (MFS) are membrane-spanning proteins that facilitate the translocation of diverse substrates including sugars, ions, amino acids, and many other molecules between the cytoplasm and the extracellular region (Law et al. 2008). The transporters can be found in all living species (for an overview of classification and comprehensive information, please refer to http://www.tscd.org (Saier et al. 2006)). The proteins of the MFS share a general three-dimensional (3D) topology, consisting of two domains that surround a substrate-transport channel also referred to as the substrate-translocation pore (reviewed in Law et al. (2008)). Owing to their ubiquitous presence and importance, dysfunctions of members of the MFS are related to several diseases, e.g. mutations of the D-glucose transporter (GLUT1, SLC2A1) cause the GLUT1 deficiency syndrome (Pascual et al. 2004), GLUT4 (SLC2A4) mutations are associated with non-insulin-dependent diabetes mellitus (Watson \& Pessin 2001), and mutations of the monocarboxylate transporter 8 (MCT8) are the underlying cause of a severe X-linked psychomotor retardation, known as the Allan-HerndonDudley syndrome (Friesema et al. 2004).

MCT8 translocates iodothyronines (Friesema et al. 2003, Kinne et al. 2010). These molecules, acting as hormones, play multi-faceted and major roles in vertebrate growth and metabolism, and are also of great importance for neuronal cell growth and development (Friesema et al. 2006, 2010, 2012, Visser et al. 2008, James et al. 2009, Bernal 2011). A vast number of pathogenic mutations of MCT8 have been identified (reviewed in Visser et al. (2011)), comprising insertions, deletions, single side-chain substitutions, and splice site variants (Friesema et al. 2010). The affected patients primarily suffer from mental retardation, severe muscle hypotonia, and, to a greater extent, are unable to speak or walk (Schwartz et al. 2005). However, there may be individual differences in the observed phenotypes (Supplementary Materials and methods and Supplementary Table 1, see section on supplementary data given at the end of this article). The molecular reasons for this phenomenon remain the subject of intense research (Visser et al. 2011).

One of the potentially modulated properties of MCT8 arising from a mutation is oligomeric organization, which may have an influence on the functionality of this transporter. Results from previous studies have indicated (Biebermann et al. 2005) that MCT8 occurs as dimers comprising two interacting protomers, or, presumably, as higher order complexes such as tetramers (Visser et al.
2009). In support of this, other members of the MFS have also been reported to form oligomers, including the glycine transporter (Bartholomaus et al. 2008), organic cation transporter 1 (Keller et al. 2011), $\gamma$-amino butyric acid transporter 1 (Korkhov et al. 2004), multidrugtransporter EmrD (Yin et al. 2006), dopamine transporter (Hastrup et al. 2001), and serotonin transporter (Kilic \& Rudnick 2000). Higher-order complexes can be functionally relevant such as those found for amphetamine transporters, for which influx and efflux occurs via separate but interacting transporter protomers (Seidel et al. 2005).

The primary goal of the current study was to examine pathogenic mutations of MCT8 pertaining to their influence on the capacity of MCT8 to form dimeric or oligomeric constellations. For this purpose, we tested 14 known pathogenic variants with partial or complete loss-of-function with regard to their capacity to form dimeric complexes in comparison with WT. Mutations were distributed over the entire transmembrane region of MCT8. Indeed, mutations were identified that alter the dimerization state of MCT8, with unexpected implications for the structural prerequisites of transporter interactions and regulation.

\section{Material and methods}

\section{Plasmid constructs}

For plasmid constructs, the expression vector pcDNA3 was used containing the human MCT8 WT cDNA or MCT8 sequences with the mutations: ins189I (insertion of isoleucine between positions 188 and 189), S194F, A224V, del230F (deletion of phenylalanine 230), ins236V (insertion of valine between positions 235 and 236), V235M, R271H, L434W, L471P, G558D, and L568P. The point mutations A224T, R445C, and L512P were introduced into the cDNA of MCT8 using the QuikChange SiteDirected Mutagenesis protocol (Agilent Technologies, La Jolla, CA, USA) and confirmed by direct sequencing. In addition, a hemagglutinin-tag (YPYDVPDYA, N-HA) at the amino-terminal end or a FLAG-tag (DYKDDDDK, C-FLAG) at the carboxy-terminal end of human MCT8 WT, as well as of the mutants, was inserted.

For the bimolecular fluorescence complementation assay, fragments of yellow fluorescent protein (YFP) were fused at the C-terminal end to the MCT8 WT and MCT8 mutant cDNA (A224T and del230F) in accordance with the method described by Nyfeler et al. (2005).

Published by Bioscientifica Ltd. 
The pcDNA3 (MCT8 and mutants) constructs were kindly provided by Dr Ulrich Schweizer (Rheinische Friedrich-Wilhelms-Universität Bonn, Germany), and the pcDNA3 (fragment-YFP1 and fragment-YFP2) constructs were provided by Dr Hans-Peter Hauri (University of Basel, Switzerland).

\section{Cell culture}

Transiently transfected COS-7 cells (an African green monkey kidney epithelial cell line), cultured in DMEM (Biochrom AG, Berlin, Germany), supplemented with 10\% fetal bovine serum (FBS), $100 \mathrm{U} / \mathrm{ml}$ penicillin, $100 \mu \mathrm{g} / \mathrm{ml}$ streptomycin, and $2 \mathrm{mM}$ L-glutamine were used in dimerization studies of the pathogenic MCT8 mutants.

\section{Investigation of dimerization of $M C T 8$ via sandwich ELISA}

To investigate dimerization of MCT8, a sandwich ELISA was performed with N-HA and C-FLAG-tagged WT MCT8 and mutants as described previously (Biebermann et al. 2005). MCT8 mutants were compared with the co-transfection of N-HA-WT and C-FLAG-WT (positive control). N-HA-WT transfection alone was used as a negative control (CTRL). In brief, 600000 COS-7 cells were seeded in $6 \mathrm{~cm}$ dishes and transiently transfected with $3 \mu \mathrm{g}$ DNA using $6 \mu \mathrm{l}$ Metafectene. At 72-h post-transfection, cells were washed, harvested, and solubilized in $200 \mu \mathrm{l}$ lysis buffer containing $10 \mathrm{mM}$ Tris- $\mathrm{HCl}$ of $\mathrm{pH} 7.4,150 \mathrm{mM} \mathrm{NaCl}, 1 \mathrm{mM}$ EDTA, $1 \mathrm{mM}$ dithiothreitol (DTT), $1 \%$ sodium-deoxycholate, $1 \%$ Nonidet P-40, and $0.2 \mathrm{mM}$ phenyl-methyl-sulfonyl fluoride overnight at $4{ }^{\circ} \mathrm{C}$. Ninety-six-well plates were coated with monoclonal anti-FLAG-M2 antibody $(10 \mu \mathrm{g} / \mathrm{ml}$ in $0.15 \mathrm{M}$ sodium tetraborate, $\mathrm{pH} 8$; SigmaAldrich) overnight at $4{ }^{\circ} \mathrm{C}$. After blocking with $10 \%$ FBS in DMEM for $1 \mathrm{~h}$ at room temperature, the plates were washed twice with $1 \times$ PBS containing 0.05\% Tween-20 (PBS-T). Following removal of cell debris, the cell lysates were incubated in the FLAG-antibody-coated 96-well plates in triplicates overnight at $4{ }^{\circ} \mathrm{C}$. The plates were washed four times with PBS-T, followed by the addition of a biotinlabeled monoclonal anti-HA antibody $(1 \mu \mathrm{g} / \mathrm{ml}$ in PBS-T; Roche) and incubation for $2 \mathrm{~h}$. The plates were washed again four times with PBS-T and incubated with peroxidaseconjugated streptavidin $(0.2 \mu \mathrm{g} / \mathrm{ml}$ in PBS-T; BioLegend, London, UK) for $1 \mathrm{~h}$. After a final washing step (four times with PBS-T), $\mathrm{H}_{2} \mathrm{O}_{2}$ and $o$-phenylendiamine ( $2.5 \mathrm{mM}$ each in $0.1 \mathrm{M}$ phosphate-citrate buffer, $\mathrm{pH} 5.0$ ) were used as substrate and chromogen. The enzyme reaction was stopped by the addition of $1 \mathrm{M} \mathrm{HCl}$ containing $0.05 \mathrm{M}$
$\mathrm{NaSO}_{3}$. The color intensity was measured at $492 \mathrm{~nm}$ and normalized to the absorbance values at $620 \mathrm{~nm}$. The readings were normalized to the protein concentrations of the cell lysates as measured with the BCA Protein Assay Reagent Kit (Thermo Fisher Scientific, Rockford, IL, USA).

It should be noted that the detection of dimers in the sandwich-ELISA approach is limited by the fact that only the combination of N-HA with C-FLAG MCT8 can be determined. The formation of N-HA/N-HA MCT8 in addition to C-FLAG/C-FLAG MCT8 also occurs but cannot be detected.

\section{Statistical analyses}

In order to investigate the significant differences between the dimerization of the differently tagged WT as a positive control and the mutants, an unpaired two-tailed Student's $t$-test with Welch's correction was performed. Statistical analyses were performed using GraphPad Prism version 5.00 for Windows (GraphPad Software, San Diego, CA, USA).

\section{Confocal microscopy of MCT8 dimers}

The YFP-based protein fragment complementation assay (PCA; Nyfeler et al. 2005) was used to confirm decreased and increased dimerization of mutants of MCT8 and their trafficking pathways in expressing COS-7 cells. In this assay, YFP is split into two halves. One half is fused to one МCT8 protomer and the other half to the other MCT8 protomer. Upon dimerization, the two halves of YFP reconstitute, forming a functioning YFP which then can be detected. Fluorescent YFP was reconstituted with regard to the dimerization of MCT8-YFP1 and -YFP2 constructs, i.e. fluorescence indicated the formation of productive and stable dimers.

COS-7 cells were seeded into six-well plates (300 000 cells/well) on poly-L-lysine-coated cover glasses and transiently transfected with $4 \mu \mathrm{l}$ Metafectene and $1.5 \mu \mathrm{g}$ DNA (MCT8-YFP1 and MCT8-YFP2)/well. Approximately $24 \mathrm{~h}$ after transfection, cells were fixed with DAPImethanol solution $(1 \mu \mathrm{g} \mathrm{DAPI} / \mathrm{ml})$ for $10 \mathrm{~min}$ at $37^{\circ} \mathrm{C}$ and mounted with Vectashield Hard Set (Vector Laboratories, Burlingame, CA, USA) on microscope slides. The cells were observed under a Zeiss LSM 510 META laser scanning microscope equipped with argon and heliumneon lasers (Carl Zeiss GmbH, Jena, Germany). The optical sections were obtained with a pinhole setting of 1 Airy unit at a resolution of $1024 \times 1024$ pixels, and the images were further analyzed using LSM 510 Software, release 3.2 (Carl Zeiss GmbH).

Published by Bioscientifica Ltd. 


\section{Molecular homology modeling of MCT8 and a potential mode of substrate binding}

A new structural human MCT8 homology model was generated by using the same general procedure as described previously (Kleinau et al. 2011), with a few significant modifications. Generally, this model is based on a structural transporter template in an 'inward-facing' conformation, namely the crystal structure of the glycerol-3-phosphate transporter (GlpT) (PDB code 1PW4 (Huang et al. 2003)). In contrast to our previous homology models, we refined the orientations of the loop regions and side-chains by extended molecular dynamic simulation. In addition, the structural template used indicated that helix 12 of MCT8 might be constituted by residues between Ala553 and Asp589. In accordance with the crystal structures of other members of the MFS, such as lactose permease (PDB code 1PV6 (Abramson et al. 2003)), or the recently solved 'outwardfacing' and substrate bound conformation of a glucose transporter (PDB code 4GBZ (Sun et al. 2012)), this helix is shorter and is regular up to the position corresponding to MCT8 amino acid position Pro572, followed by a loop region that continues with a short helix. Moreover, this small helical section interacts with further transporter regions, as has been observed for other crystal structures, and these might constitute stabilizing interactions (Sun et al. 2012). Therefore the MCT8 helix 12 was constructed in accordance with these structural features and terminates with a regular helix conformation at Pro572, whereby adjacent residues are allocated close to the intracellular loops.

By fixing the backbone atoms, molecular dynamics ( $4 \mathrm{~ns}$ ) were subjected to the flexible amino acid side-chains of the model (Sybyl-X2.0, Certara, Princeton, NJ, USA). The entire system was then minimized by fixing the backbone atoms of the transmembrane helices (TMHs) until convergence at a termination gradient of $0.05 \mathrm{kcal} /$ mol $\times \AA$. This procedure was repeated thrice. Finally, the model was minimized without constraints using the AMBER 7.0 force field. Structure images were produced using the PyMOL Molecular Graphics System, version 1.3 (Schrödinger, LLC, New York, NY, USA).

The resulting structural model was utilized to demonstrate the positions of mutations, visualize the substrate translocation channel (Fig. 1A, B and C), and also docking of T3 into MCT8 (Fig. 1D). Substrate docking (details of the docking procedure are described in the Supplementary Material) was performed to evaluate the overlap between spatial regions important for substrate binding and positions of pathogenic mutations with a potential effect on oligomerization.

\section{Results}

The intention of this study was to unravel the question of whether naturally occurring mutations in MCT8 might have an effect on formation of MCT8 dimers. Fourteen known naturally occurring mutations, which have been identified in affected patients, were chosen. These mutations are distributed over the entire transporter structure and they are mostly characterized by a singleside-chain substitution. These 14 variants constitute the main fraction of known naturally occurring single-sidechain substitutions. Exon deletions or stop-codon variants were not explored. For seven of these variants of MCT8 (ins189I, A224V, R271H, L471P, L512P, G558D, and L568P), dimer assembly was identical to that of WT MCT8 dimers as determined by sandwich ELISA (Table 1 and Fig. 2). However, modifications of the dimerization ability were detected for seven variants of MCT8 (S194F, A224T, del230F, V235M, ins236V, L434W, and R445C).

\section{Substrate uptake of WT and mutant variants of MCT8 in COS-7 cells}

Extensive functional in vitro characterization of MCT8 mutants revealed that the results obtained might vary depending on the cellular system used (Jansen et al. 2005, 2008, Kinne et al. 2009, Capri et al. 2013, Kersseboom et al. 2013). This therefore plays a decisive role in why a precise established relationship does not exist between the genotype, molecular characteristics of MCT8 variants (localization, transport routes), and the severity of patient phenotypes (Visser et al. 2011) (summarized in Supplementary Table 1). The functional characteristics of the MCT8 mutants investigated in this current study were previously explored by in vitro analyses using JEG, MDCK, or COS cells (Schwartz et al. 2005, Jansen et al. 2008, Kinne etal. 2009, 2010, Capri et al. 2013, Visser et al. 2013, Groeneweg et al. 2014). The cellular localization of MCT8 mutants and the rates of thyroid hormone uptake for all of these mutants finally revealed a diminished rate of $\mathrm{T} 3$ uptake.

However, we analyzed COS-7 cells for their suitability for the functional characterization of MCT8 mutants with respect to T3 uptake. The T3 transport function of six particular pathogenic variants of MCT8 (S194F, A224T, del230F, V235M, L471P, and L568P) was confirmed to be reduced in comparison with WT (Supplementary Figure 1, see section on supplementary data given at the end of this article). This provides evidence that COS-7 cells are capable of expressing functional WT MCT8 (Visser et al. 2009, Kersseboom et al. 2013), and are a useful system for MCT8 dimerization studies

Published by Bioscientifica Ltd. 
A

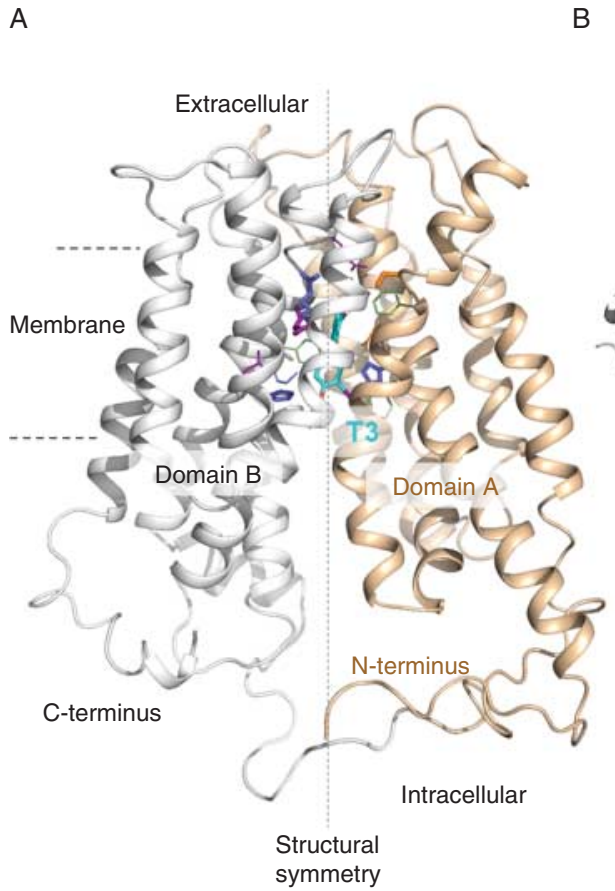

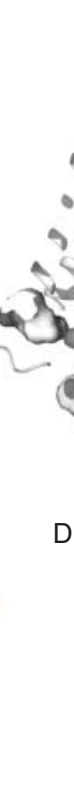

ba $c$

C Top-view

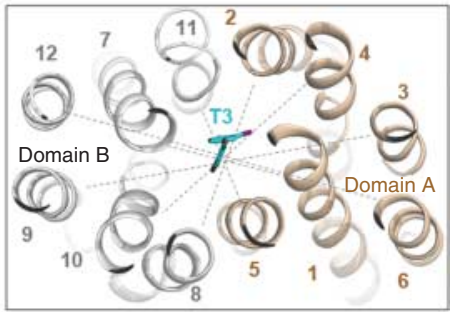

\section{Figure 1}

Structural MCT8 model in an inward-facing conformation occupied by substrate. This MCT8 homology model (backbone ribbon) represents a potential 3D-structure of MCT8 that is subdivided into two halves: domain A (beige) and domain B (white). The $\mathrm{N}$ - and C-termini are localized intracellularly and each domain is constituted by six helices that are in a mirror-like arrangement ( $A$ ) and with center-axis symmetry (dashed line). In addition, we propose a specific binding mode of $\mathrm{T} 3$ inside the substratetranslocation channel (B), with particular amino acids participating in the interaction with $\mathrm{T} 3$ (cyan sticks). This putative docking position is based on a combination of structural/functional data and results from bioinformatic procedures (see Materials and methods section in the Supplementary Material). $T 3$ is most probably bound between the two domains ( $B$, inner surface representation), exactly at the symmetry axis between the domains and helices (C). Details of putative MCT8/T3 interactions are represented in D. Hydrophilic and charged amino acids interact with the carboxy group of T3, whereby the positively charged side-chain of Arg445 might be a key player. The negatively charged side-chain of Glu422 participates in binding of T3 close to the bottom of the substrate translocation channel in this conformation, and also presumptively interacts with $\mathrm{Arg} 445$ (H-bonds

by western blotting (Visser et al. 2009, Kersseboom et al. 2013) and sandwich ELISA (Biebermann et al. 2005).

\section{Specific naturally occurring mutations inhibit dimerization of MCT8}

Modifications of the dimeric state of specific MCT8 mutants were detected: S194F in TMH1, A224T in TMH2, L434W (ECL4/TMH8) and R445C in TMH8 were observed to result shown as dotted yellow lines). Both residues are conserved within the MCT group. Our newly generated model indicates that Asp498 and Lys418 should function as counteracting charged residues, whereby interactions with the substrate (intermediates) are assumed. Moreover, in this arrangement, the two histidines His 192 and His415 participate in substrate transport. On the basis of the occurrence of pathogenic mutations and results from bioinformatic studies (Friesema et al. 2010, Kinne et al. 2010, Kleinau et al. 2011), histidine 192 has previously been suggested to be of great importance for substrate translocation, as has been recently confirmed by the results of experimental in vitro studies (Braun et al. 2013, Groeneweg et al. 2013). In addition, His415 has also been predicted (Kleinau et al. 2011) and confirmed to be a determinant of substrate translocation in MCT8 (Braun et al. 2013). Further residues that may participate in this proposed T3-binding pocket are amino acids with hydrophobic and aromatic side-chain properties, such as lle534, Phe189, Phe316, or Tyr419. They cover and adjust the substrate, as is also observed for those residues in crystal structures of T3-binding proteins (see also Supplementary Material). In addition, Ser317, Asn193, and Glu426 should make significant contributions to parameters of binding, translocation, and substrate selectivity.

in a reduced level of dimerization by $30-60 \%$ in comparison with WT mutants (Table 1 and Fig. 3). The A224T mutation led to a reduction in dimerization capability to form oligomers, by approximately $65 \%$, while the $\mathrm{R} 445 \mathrm{C}, \mathrm{S} 194 \mathrm{~F}$, and L434W mutations resulted in 53, 45, and $36 \%$ reductions in dimerization capability in comparison with WT, respectively, as assessed by sandwich ELISA.

Interestingly, at position Ala224, two amino acid substitutions altered dimeric formation differentially.

Published by Bioscientifica Ltd 
Table 1 Spatial localization of MCT8 mutations in the protein, and effects on dimerization. The mutations analyzed here are located in different helices, or transitions between helices and loops. Besides the regular position number (starting from the startcodon position 1) for each mutation, the number in accordance with the unifying MCT-group numbering scheme is also provided (Kinne et al. 2010), in order to enable comparison between different members of the MCT group. This position identifier scheme uses a highly conserved residue in each TMH as a common reference for all members of the MCT family. The first number is related to the particular helix, the second number after the dot determines the specific position in relation to the conserved residue. For example, the highly conserved proline in $\mathrm{TMH} 2$ is defined as 2.50 and the highly conserved glycine from $\mathrm{TMH} 3$ is defined as 3.50 . The nomenclature follows the standard conventions of the Human Genome Variation Society (HGVS)

\begin{tabular}{l}
$\begin{array}{l}\text { Mutation } \\
\text { (nomenclature of HGVS) }\end{array}$ \\
\hline p.Ile188_Phe189inslle \\
p.Ser194Phe \\
p.Ala224Val \\
p.Ala224Thr \\
p.Phe230del \\
p.Val235Met \\
p.Val235_Ser236insVal \\
p.Arg271His \\
p.Leu434Trp \\
p.Arg445Cys \\
p.Leu471Pro \\
p.Leu512Pro \\
p.Gly558Asp \\
p.Leu568Pro
\end{tabular}

\begin{tabular}{c}
$\begin{array}{c}\text { Abbreviation used } \\
\text { in this study }\end{array}$ \\
\hline ins189I \\
S194F \\
A224V \\
A224T \\
del230F \\
V235M \\
ins236V \\
R271H \\
L434W \\
R445C \\
L471P \\
L512P \\
G558D \\
L568P
\end{tabular}

\begin{tabular}{|c|}
\hline $\begin{array}{c}\text { Dimerization compared } \\
\text { with WT }\end{array}$ \\
\hline$=$ \\
\hline- \\
\hline$=$ \\
\hline- \\
\hline+ \\
\hline+ \\
\hline+ \\
\hline$=$ \\
\hline- \\
\hline- \\
\hline$=$ \\
\hline$=$ \\
\hline$=$ \\
\hline$=$ \\
\hline
\end{tabular}

$\begin{gathered}\text { Structural } \\ \text { localization }\end{gathered}$
TMH1
TMH1
TMH2
TMH2
TMH2
TMH2
TMH2
ECL2/TMH4
ECL4/TMH8
TMH8
TMH9
TMH10/ICL5
TMH12
TMH12

\begin{tabular}{rr}
$\begin{array}{c}\text { Unifying } \\
\text { number }\end{array}$ \\
\hline 1.64 \\
1.69 \\
2.41 \\
2.41 \\
2.47 \\
2.52 \\
2.53 \\
4.39 \\
8.39 \\
8.50 \\
9.49 \\
10.63 \\
12.54 \\
12.64
\end{tabular}

Four mutants are characterized by diminished $(-)$ capacity to homo-dimerize compared with the WT. Three mutations in TMH2 lead to increased dimerization $(+)$ ' $^{\prime}={ }^{\prime}$ Indicates equal to WT. TMH, transmembrane helix; ECL, extracellular loop; ICL, intracellular loop.

The A224V variant formed homo-dimeric complexes similarly to the WT, whereas in mutant A224T, the structure of MCT8 was mostly monomeric.

The WT positions of the four dimerization altering amino acids S194, A224, L434, and R445 were mapped onto the MCT8 model and revealed a spatial localization directly at or in close proximity to the transport channel cavity ('inward-facing' conformation; Figs 1 and 4). This finding is in contrast to the hypothesis that only substitutions at the protein surface disrupt the protomer-protomer contacts.

\section{Mutations in TMH2 led to an increased capacity for dimerization}

Surprisingly, we detected increased dimerization capabilities in the variants of MCT8 with the del230F, V235M, and ins236V mutations (Table 1 and Fig. 3). The increase in dimers detectable by sandwich ELISA amounted up to $150-175 \%$ when compared with WT values, i.e. del230F reached 148\%, V235M 151\%, and ins236V 174\%.

According to our structural MCT8 model, amino acid substitutions, deletions, or insertions, causing increased MCT8 dimerization (Table 1 and Fig. 4) are located exclusively in TMH2 of the WT protein and thus outside of the transport channel cavity. Insertions or deletions modify the orientation of this specific helical section close to the intracellular site. It is noteworthy that the sidechain at position 235 is without any intramolecular interaction partner in this monomeric MCT8 model and substitution of V235M, therefore, does not alter any sidechain interactions. However, all three mutations indicate that this structural section should be of great importance in the formation of higher-order complexes.

\section{Investigation of WT and mutant MCT8 by bimolecular complementation and confocal laser scanning microscopy analysis}

To verify data obtained with the sandwich-ELISA approach, we choose one MCT8 variant from the group with increased dimerization (del230F) and one characterized by decreased dimerization (A224T). Dimerization of WT and of A224T or del230F MCT8 proteins was analyzed using a bimolecular fluorescence complementation assay. In this assay, fluorescence is only detectable upon dimer formation.

The cells expressing MCT8 WT dimers (Fig. 5A, B and C) appeared evenly distributed and firmly adherent to the cover glasses, while fluorescence was detectable in reticular structures reminiscent of the endoplasmic

Published by Bioscientifica Ltd. 


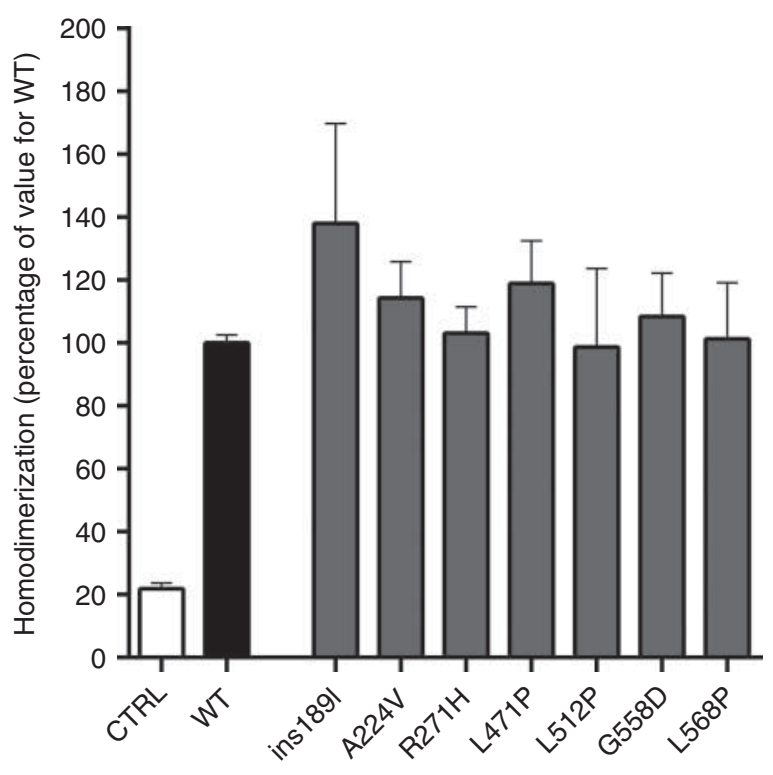

Figure 2

MCT8 mutants with a capacity for homodimerization similar to that of the WT. The capacity for dimerization was tested by sandwich ELISA. COS-7 cells were transiently co-transfected with N-HA and C-FLAG WT MCT8 (MCT8 WT) or mutant MCT8. Single transfection of N-HA-tagged WT served as a negative control (CTRL, white column). No significant differences compared with the MCT8 WT could be shown for the mutations ins189l, A224V, R271H, L471P, L512P, G558D, and L568P (grey columns). The mean absorption $(492 \mathrm{~nm} / 620 \mathrm{~nm})$ was normalized to $\mathrm{mg} / \mathrm{ml}$ protein concentration and given as percentage of WT/WT homodimer formation (black column, absorption (492/620)/mg/ml of protein: $0.27 \pm 0.6)$. Data are depicted as means + S.E.M. from three independent experiments carried out in triplicate.

reticulum, in vesicles (arrows), and at the cell surface (arrowheads). In addition, approximately $50 \%$ of cells expressing WT dimers exhibited small foci of aggregated, strongly fluorescent protein distributed throughout the cytoplasm.

Likewise, cells forming dimers of the del230F (Fig. 5D, $\mathrm{E}$ and $\mathrm{F}$ ) mutant were adherent and disseminated on the substratum displaying fluorescence in reticular and vesicular structures (arrows), as well as at the cell surface (arrowheads). In comparison with WT dimers, del230Fmutant expressing cells contained numerous and smaller foci of fluorescent dimeric or oligomeric proteins, which were often observed to have a bead-on-a-string appearance, which is expected for transport vesicles in transit from the Golgi apparatus to the cell surface (Fig. 5D). These findings are in accordance with the sandwich-ELISA data, thus indicating an enhanced dimerization capability compared with WT MCT8.

In stark contrast, cells expressing the A224T dimeric proteins (Fig. 5G, H and I) were less evenly distributed on the substratum, with a curved shape and fewer cellular extensions in comparison with WT dimers and del230F dimer-expressing cells. Moreover, COS-7 cells expressing the A224T variant of МCT8 were characterized by the presence of prominent foci containing fluorescent protein which accumulated in the peri-nuclear regions, which are characteristic of the so-called aggresomes that are typically formed as a consequence of protein misfolding and overload of the ER-associated degradation machinery. Therefore, the formation of A224T dimers at the cell surface of expressing COS-7 cells is less frequent than in cells expressing WT МСT8 or the del230F variant. Results obtained using confocal laser scanning microscopy thus indicate a potential association with results obtained with the sandwich-ELISA method; however, we cannot rule out the possibility that aggresomes identified by laser scanning microscopy could be detected using the sandwich-ELISA approach.

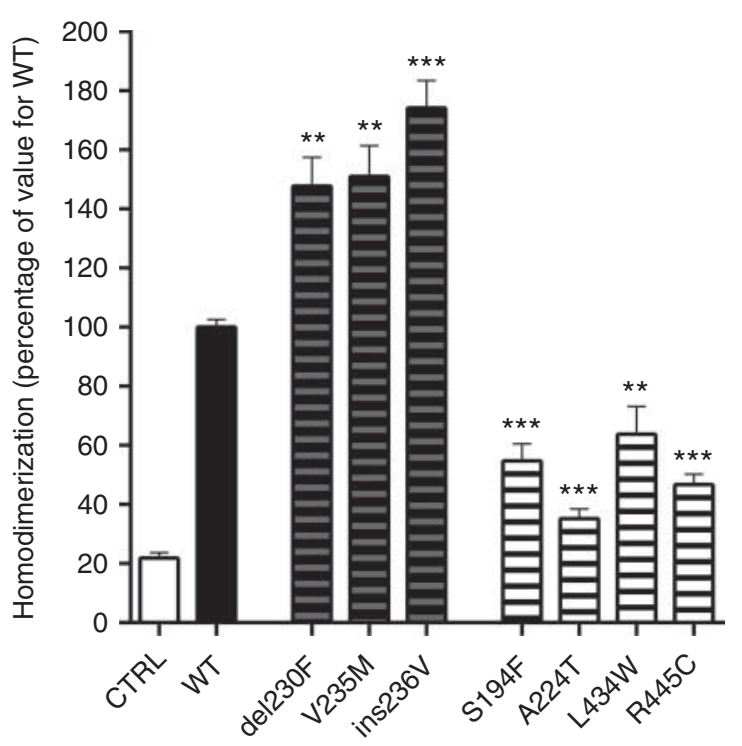

Figure 3

Modulation of MCT8 homodimerization by pathogenic mutations. The capacity of mutated $M C T 8$ transporters to form dimers compared with MCT8 WT was measured using a sandwich-ELISA approach. N-HA-tagged MCT8 WT is defined as the negative control (CTRL, white column). The co-transfection of N-HA-tagged MCT8 WT and C-FLAG-tagged MCT8 WT serves as a positive control (black column). The mutations del230F, V235M, and ins236V increased dimerization of MCT8 (grey columns with black lines), whereas mutations S194F, A224T, L434W, and R445C (white columns with black lines) diminished the capacity for dimerization. The mean absorption $(492 \mathrm{~nm} / 620 \mathrm{~nm}$ ) was calculated per $\mathrm{mg} / \mathrm{ml}$ protein of transiently transfected COS-7 cells and shown as a percentage of the value for the WT/WT homodimer (black column, absorption (492/620)/ $\mathrm{mg} / \mathrm{ml}$ of protein: $0.27 \pm 0.6$ ). Data were assessed from three independent experiments, each performed in triplicate and represent mean + s.E.M. Statistical significance, as assessed by unpaired $t$-test with Welch's correction, is denoted as $* * P<0.01$ and $* * * P<0.001$.

Published by Bioscientifica Ltd 


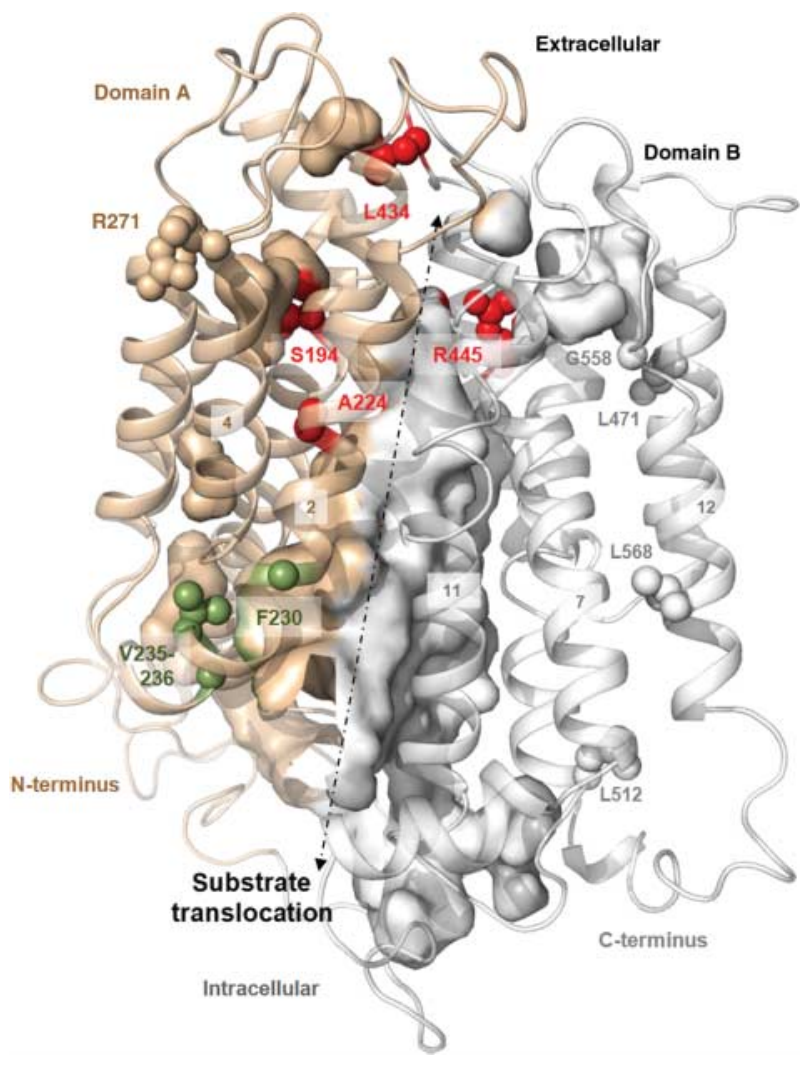

Figure 4

Positions of investigated pathogenic mutations in a three-dimensional model of MCT8. The positions of the pathogenic substitutions investigated here (atom spheres) are mapped onto the structural MCT8 model (white backbone ribbon and inner surface of the transport channel). Substitutions inhibiting MCT8 oligomerization are indicated in red and are localized close to the middle section of the transport channel between domains $A$ and $B$. It is assumed that during transport of substrate, this 'bottle-neck' of the closed channel is opened by helical re-arrangements. A few of these WT amino acids are known to be involved in both substrate transport and intramolecular interaction, such as Arg445 (Kinne et al. 2010, Capri et al. 2013). Therefore, the tri-functionality of particular WT amino acids is important for: i) binding of substrate, ii) participating in intramolecular interaction network(s), and iii) switching the global protomer conformation. Notably, while a few mutations do not modify oligomerization of MCT8 (white spheres), three MCT8 mutations lead to an increase in oligomerization, and are localized exclusively at a specific section of $\mathrm{TMH} 2$ (green spheres). Insertion, deletion, or residue substitution between positions 230 and 236 increased the capacity for dimerization. The increase in oligomeric MCT8 molecules as a result of modifications at TMH2 might be related to optimization of a shape-fit of two MCT8 protomers to one another, most probably in close vicinity to this specific МCT8 region.

\section{Discussion}

In the current study, pathogenic mutations leading to partial monomerization of MCT8 were identified (S194F, A224T, L434W, and R445C). Specific mutants (del230F, V235M, and ins236V) with an increased level of dimerization, and oligomerization, were also found
(Fig. 3). Interestingly, the R445C (Groeneweg et al. 2014) and ins236V (Kersseboom et al. 2013) variants of MCT8 have been examined in COS cells, using approaches which included analyses of their dimerization capacity and cellular distribution. In accordance with our findings, the western blotting data presented in previous studies (Kersseboom et al. 2013, Groeneweg et al. 2014) might also indicate increased (ins236V) or decreased (R445C) dimerization of these MCT8 variants.

\section{Transporter oligomers and structure-function relationships}

It is known from studies of G-protein-coupled receptors (GPCRs) that an equilibrium may exist between monomers and oligomers (Lambert 2010, Teichmann et al. 2012, Calebiro et al. 2013), implying that protomer-protomer interfaces are flexible or transient. Amino acid mutations can shift this equilibrium toward a monomeric or more oligomeric state if they modify the interface(s) between the protomers.

It remains unknown how this protomer-protomer interaction is constituted for MCT8. Cysteine residues that are potentially disulfide bridged do not participate in the structure of putative MCT8 protomer interface(s) in oligomers (Visser et al. 2009), as has been reported, for example, for GPCRs (Berthouze et al. 2007) and other members of the MFS (Hastrup et al. 2003, Henriksen et al. 2005). Moreover, it has been demonstrated that the amino acids from positions 267 to 360 (putative TMH4-6) are not involved in MCT8 protomer contacts (Visser et al. 2009). However, several contact regions for membrane-spanning transporter oligomers have already been reported (oligomerization is inhibited by mutation): the bacterial $\mathrm{Ca}^{2+} / \mathrm{H}^{+}$antiporter (Ridilla et al. 2012) - contacts at TMH2 and TMH6; the $\mathrm{Na}^{+} / \mathrm{H}^{+}$antiporter NhaA (Gerchman et al. 2001) - loop transition on the cytoplasmatic side of helix 8; the dopamine transporter (Hastrup et al. 2003) - at the extracellular end of TMH6; the $\gamma$-amino-butyric acid transporter 1 - at TMH2 (Korkhov et al. 2004); and the glycine transporter 2 (GlyT2) extracellular transition of TMH6. Very recently, TMH2 has been identified as a crucial dimerization interface of the bacterial sweet transporter (Xu et al. 2014).

It is of note that, taking these insights into dimerconstitution, different interfaces of MFS members, and our findings on MCT8 under consideration, it might be also feasible that truncated or mutated (non-functional) variants of MCT8 interact with other membrane proteins such as further members of the MFS family. This should

Published by Bioscientifica Ltd. 

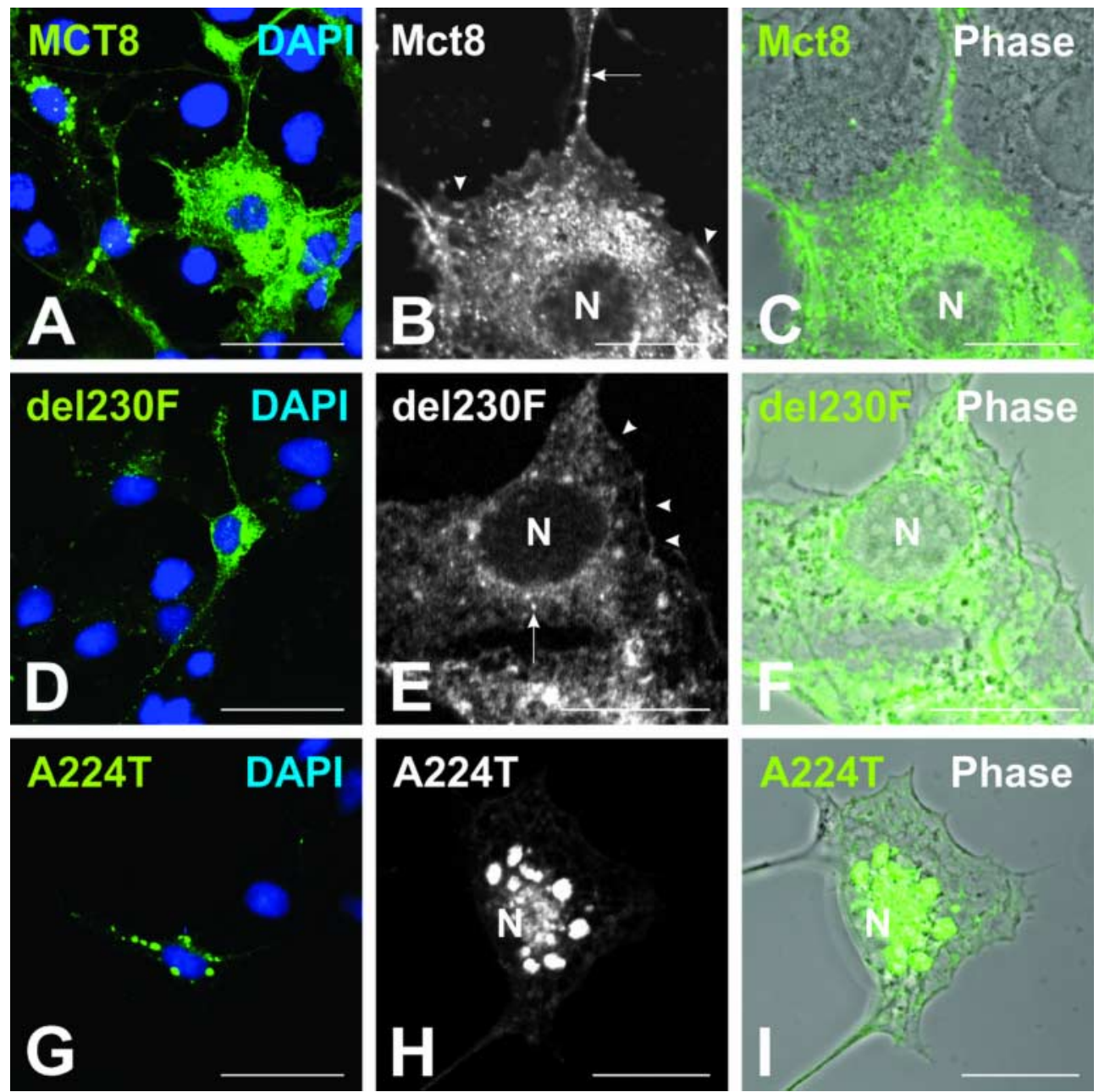

Figure 5

Distribution of MCT8 dimers in COS-7 cells. Confocal laser scanning micrographs of COS-7 cells expressing MCT8 WT (A, B and C), the del230F ( $D, E$ and $F$ ), and the A224T ( $G, H$ and $I$ ) dimers are shown as multiple- (A, D and $G$ ) or single-channel fluorescence micrographs (B, E and $H)$, or depicted as overlays with the corresponding phase-contrast images ( $\mathrm{C}, \mathrm{F}$ and I). Fluorescence indicates bimolecular complementation through dimerization of MCT8-YFP1 and MCT8-YFP2 fragments (green signals in A, C, D, F, $G, I$ and white signals in $B, E, H$ ). Nuclei were counter-stained with DAPI (cyan signals in $A, D$ and $G$ ). Cell-surface and vesicular occurrence of

theoretically lead also to dominant-negative effects on interacting WT proteins.

Notably, the protomer-protomer contacts for those transporters are spatially located at the protein surface in order to establish intermolecular contacts. In contrast, in this current study, the identified mutations associated with inhibition of dimerization are located close to the putative substrate-translocation channel in the center of MCT8. In addition, mutations in TMH2 of MCT8 are surface-directed and increase the capacity for oligomerization. A number of explanations could be put forward for oligomerized transporter molecules are indicated by arrows and arrowheads respectively ( $\mathrm{B}$ and $\mathrm{E}$ ). Note the well-spread phenotype of COS-7 cells expressing MCT8 WT and del230F oligomers in the rough endoplasmic reticulum, the peri-nuclear Golgi-apparatus, and in numerous vesicles in transit to and from the plasma membrane (A, B, C, D, E and F). In contrast, the A224T dimers revealed an altered morphology and featured large aggregates accumulating in the peri-nuclear region, resulting from the dense packing of the mis-folded transporter mutant forms. $\mathrm{N}$ denotes nuclei, scale bars represent $50 \mu \mathrm{m}$ in $\mathrm{A}, \mathrm{D}, \mathrm{G}$ and $20 \mu \mathrm{m}$ in B, C, E, F, H, I.

these findings. In order to adequately address this, the thus far proposed molecular transport mechanisms at MCT8 will be briefly described: for transporter monomers, it is assumed that substrate binding triggers the transition between 'outward-facing' and 'inward-facing' conformations by a so-called 'rocker-switch' mechanism (rocking back and forth around a central axis), whereby the structure of the transporter undergoes global structural rearrangements (Huang et al. 2003, Yin et al. 2006, Law et al. 2008, Sun et al. 2012). Following substrate binding, the two transporter domains A and B (Fig. 1) move closer,

Published by Bioscientifica Ltd. 
either intracellularly or extracellularly, respectively. This process is supported by flexibility in specific helices caused by irregular helical conformations. The global movement (rocking back and forth) is triggered by substrate binding between the domains that weakens or/and increases specific intramolecular (inter-helical) interactions. As a consequence, mutations in the transport channel (with sensitivity for substrate binding) are also likely to have a dramatic effect on the transporter conformation, because they are localized at predestined positions to initiate global structural shifts. These conformational shifts induced in the transport channel of protomers could also modify the oligomer organization by indirectly altering the interfaces between the (modified) protomers.

But how should this relate to the function of the transporters? It is known that transporters can function by distinct mechanisms, such as i) uniporters, which transport only one type of substrate (substrate-gradient regulated); ii) antiporters, which transport two or more substrates but in opposite directions across the membrane, and iii) symporters, which translocate two or more substrates in the same direction simultaneously. Hence, an oligomeric transporter organization could be linked with the counter-transport of substrate(s), particularly the regulation of influx and efflux that might occur through separated but coupled protomers (Seidel et al. 2005). In a potential protomer-protomer constellation for coupled substrate influx/efflux, the two transporter molecules could have different conformations that mutually modify one another during substrate transport. As a consequence, particular mutations in the transport channel that modify the transporter-protomer conformation might also disturb the functional interplay between protomers in an oligomeric constellation.

\section{TMH2 is a key player in the function of MCT8}

Our results highlight helix 2 as a potential key player in the function of MCT8. This hypothesis is supported by the finding of mutations that increase dimerization at a specific section of TMH2 close to the intracellular site. From their location and functional data (Figs 3 and 4), we can conclude that this helical part most probably weakens the oligomeric interface - 'destabilization' - and that mutations (and their resulting structural modifications) reverse this negative effect on oligomerization.

Moreover, the alanine-to-threonine substitution at position 224 in the more central part of the protein leads to oligomer dissociation. The A224V single substitution differs from the A224T mutant and does not alter the oligomeric state (Figs 2 and 3). This indicates a fine-tuned molecular mechanism at this WT residue. Valine is hydrophobic and bulkier than alanine and will, therefore, lead to repulsion in this tightly packed vicinity close to the substrate-translocation region (inhibiting substrate transport directly or indirectly). In contrast, threonine is hydrophilic and smaller than valine, but might interact via $\mathrm{H}$-bonds with further side-chains in close proximity (like Tyr419) or with the helical backbone. In conclusion, a constraint (H-bond) at position 224 might inhibit oligomerization, whereas the flexibility of valine in a hydrophobic cage retains the capacity for oligomerization.

\section{Concluding remarks}

The results of this study indicate that there is a relationship between oligomerization and functional parameters of MCT8, but the question on an obligatory functional need for dimerization of MCT8 remains to be answered. Furthermore, on the cellular level mutant variants of MCT8 might interact differently with other ancillary proteins for proper localization at the plasma membrane and transport function. Interaction with the regulator PTTG-binding factor (PBF) has been previously demonstrated to be associated with a reduction in expression of MCT8 at the cell surface (Smith et al. 2012). Finally, our data put emphasis on the general question surrounding substrate-induced or constitutive oligomerization versus monomerization (oligomer dissociation) in members of the MFS, which has not yet been elucidated.

\section{Supplementary data}

This is linked to the online version of the paper at http://dx.doi.org/10.1530/ JME-14-0272.

Declaration of interest

The authors declare that there is no conflict of interest that could be perceived as prejudicing the impartiality of the research reported.

\section{Funding}

This work was supported by the Deutsche Forschungsgemeinschaft (DFG), projects BI 893/6-3, BI893/5-1, KL 2334/2-2, Ki 1751/1-1, MO 1018/2-1, FU 356/7-1 and 8-1, and BR 1308/11-1

\section{References}

Abramson J, Smirnova I, Kasho V, Verner G, Kaback HR \& Iwata S 2003 Structure and mechanism of the lactose permease of Escherichia coli. Science 301 610-615. (doi:10.1126/science.1088196)

Published by Bioscientifica Ltd. http://jme.endocrinology-journals.org DOI: 10.1530/JME-14-0272
C) 2015 Society for Endocrinology Printed in Great Britain 
Bartholomaus I, Milan-Lobo L, Nicke A, Dutertre S, Hastrup H, Jha A, Gether U, Sitte HH, Betz H \& Eulenburg V 2008 Glycine transporter dimers: evidence for occurrence in the plasma membrane. Journal of Biological Chemistry 283 10978-10991. (doi:10.1074/jbc.M800622200)

Bernal J 2011 Thyroid hormone transport in developing brain. Current Opinion in Endocrinology, Diabetes, and Obesity 18 295-299. (doi:10. 1097/MED.0b013e32834a78b3)

Berthouze M, Rivail L, Lucas A, Ayoub MA, Russo O, Sicsic S, Fischmeister R, Berque-Bestel I, Jockers R \& Lezoualc'h F 2007 Two transmembrane Cys residues are involved in $5-\mathrm{HT}_{4}$ receptor dimerization. Biochemical and Biophysical Research Communications 356 642-647. (doi:10.1016/j.bbrc. 2007.03.030)

Biebermann H, Ambrugger P, Tarnow P, von Moers A, Schweizer U \& Grueters A 2005 Extended clinical phenotype, endocrine investigations and functional studies of a loss-of-function mutation A150V in the thyroid hormone specific transporter MCT8. European Journal of Endocrinology 153 359-366. (doi:10.1530/eje.1.01980)

Braun D, Lelios I, Krause G \& Schweizer U 2013 Histidines in potential substrate recognition sites affect thyroid hormone transport by monocarboxylate transporter 8 (MCT8). Endocrinology 154 2553-2561. (doi:10.1210/en.2012-2197)

Calebiro D, Rieken F, Wagner J, Sungkaworn T, Zabel U, Borzi A, Cocucci E, Zurn A \& Lohse MJ 2013 Single-molecule analysis of fluorescently labeled G-protein-coupled receptors reveals complexes with distinct dynamics and organization. PNAS 110 743-748. (doi:10.1073/pnas.1205798110)

Capri Y, Friesema EC, Kersseboom S, Touraine R, Monnier A, Eymard-Pierre E, Des Portes V, De Michele G, Brady AF, Boespflug-Tanguy O et al. 2013 Relevance of different cellular models in determining the effects of mutations on SLC16A2/MCT8 thyroid hormone transporter function and genotype-phenotype correlation. Human Mutation 34 1018-1025. (doi:10.1002/humu.22331)

Friesema EC, Ganguly S, Abdalla A, Manning Fox JE, Halestrap AP \& Visser TJ 2003 Identification of monocarboxylate transporter 8 as a specific thyroid hormone transporter. Journal of Biological Chemistry $\mathbf{2 7 8}$ 40128-40135. (doi:10.1074/jbc.M300909200)

Friesema EC, Grueters A, Biebermann H, Krude H, von Moers A, Reeser M, Barrett TG, Mancilla EE, Svensson J, Kester MH et al. 2004 Association between mutations in a thyroid hormone transporter and severe $\mathrm{X}$-linked psychomotor retardation. Lancet 364 1435-1437. (doi:10.1016/S0140-6736(04)17226-7)

Friesema EC, Jansen J, Heuer H, Trajkovic M, Bauer K \& Visser TJ 2006 Mechanisms of disease: psychomotor retardation and high $\mathrm{T}_{3}$ levels caused by mutations in monocarboxylate transporter 8. Nature Clinical Practice. Endocrinology \& Metabolism 2 512-523. (doi:10.1038/ ncpendmet0262)

Friesema EC, Visser WE \& Visser TJ 2010 Genetics and phenomics of thyroid hormone transport by MCT8. Molecular and Cellular Endocrinology 322 107-113. (doi:10.1016/j.mce.2010.01.016)

Friesema EC, Visser TJ, Borgers AJ, Kalsbeek A, Swaab DF, Fliers E \& Alkemade A 2012 Thyroid hormone transporters and deiodinases in the developing human hypothalamus. European Journal of Endocrinology 167 379-386. (doi:10.1530/EJE-12-0177)

Gerchman Y, Rimon A, Venturi M \& Padan E 2001 Oligomerization of NhaA, the $\mathrm{Na}^{+} / \mathrm{H}^{+}$antiporter of Escherichia coli in the membrane and its functional and structural consequences. Biochemistry 40 3403-3412. (doi:10.1021/bi002669o)

Groeneweg S, Lima de Souza EC, Visser WE, Peeters RP \& Visser TJ 2013 Importance of His192 in the human thyroid hormone transporter MCT8 for substrate recognition. Endocrinology 154 2525-2532. (doi:10.1210/en.2012-2225)

Groeneweg S, Friesema EC, Kersseboom S, Klootwijk W, Visser WE, Peeters RP \& Visser TJ 2014 The role of Arg445 and Asp498 in the human thyroid hormone transporter MCT8. Endocrinology 155 618-626. (doi:10.1210/en.2013-1521)

Hastrup H, Karlin A \& Javitch JA 2001 Symmetrical dimer of the human dopamine transporter revealed by cross-linking Cys-306 at the extracellular end of the sixth transmembrane segment. PNAS $\mathbf{9 8}$ 10055-10060. (doi:10.1073/pnas.181344298)

Hastrup H, Sen N \& Javitch JA 2003 The human dopamine transporter forms a tetramer in the plasma membrane: cross-linking of a cysteine in the fourth transmembrane segment is sensitive to cocaine analogs. Journal of Biological Chemistry 278 45045-45048. (doi:10.1074/ jbc.C300349200)

Henriksen U, Fog JU, Litman T \& Gether U 2005 Identification of intra- and intermolecular disulfide bridges in the multidrug resistance transporter ABCG2. Journal of Biological Chemistry 280 36926-36934. (doi:10.1074/ jbc.M502937200)

Huang Y, Lemieux MJ, Song J, Auer M \& Wang DN 2003 Structure and mechanism of the glycerol-3-phosphate transporter from Escherichia coli. Science 301 616-620. (doi:10.1126/science.1087619)

James SR, Franklyn JA, Reaves BJ, Smith VE, Chan SY, Barrett TG, Kilby MD \& McCabe CJ 2009 Monocarboxylate transporter 8 in neuronal cell growth. Endocrinology 150 1961-1969. (doi:10.1210/en.2008-1031)

Jansen J, Friesema EC, Milici C \& Visser TJ 2005 Thyroid hormone transporters in health and disease. Thyroid 15 757-768. (doi:10.1089/ thy.2005.15.757)

Jansen J, Friesema EC, Kester MH, Schwartz CE \& Visser TJ 2008 Genotypephenotype relationship in patients with mutations in thyroid hormone transporter MCT8. Endocrinology 149 2184-2190. (doi:10.1210/en. 2007-1475)

Keller T, Egenberger B, Gorboulev V, Bernhard F, Uzelac Z, Gorbunov D, Wirth C, Koppatz S, Dotsch V, Hunte C et al. 2011 The large extracellular loop of organic cation transporter 1 influences substrate affinity and is pivotal for oligomerization. Journal of Biological Chemistry 286 37874-37886. (doi:10.1074/jbc.M111.289330)

Kersseboom S, Kremers GJ, Friesema EC, Visser WE, Klootwijk W, Peeters RP \& Visser TJ 2013 Mutations in MCT8 in patients with Allan-HerndonDudley-syndrome affecting its cellular distribution. Molecular Endocrinology 27 801-813. (doi:10.1210/me.2012-1356)

Kilic F \& Rudnick G 2000 Oligomerization of serotonin transporter and its functional consequences. PNAS 97 3106-3111. (doi:10.1073/pnas. 97.7.3106)

Kinne A, Roth S, Biebermann H, Kohrle J, Gruters A \& Schweizer U 2009 Surface translocation and tri-iodothyronine uptake of mutant MCT8 proteins are cell type-dependent. Journal of Molecular Endocrinology $\mathbf{4 3}$ 263-271. (doi:10.1677/JME-09-0043)

Kinne A, Kleinau G, Hoefig CS, Gruters A, Kohrle J, Krause G \& Schweizer U 2010 Essential molecular determinants for thyroid hormone transport and first structural implications for monocarboxylate transporter 8. Journal of Biological Chemistry 285 28054-28063. (doi:10.1074/jbc. M110.129577)

Kleinau G, Schweizer U, Kinne A, Kohrle J, Gruters A, Krude H \& Biebermann H 2011 Insights into molecular properties of the human monocarboxylate transporter 8 by combining functional with structural information. Thyroid Research 4 (Suppl 1) S4. (doi:10.1186/17566614-4-S1-S4)

Korkhov VM, Farhan H, Freissmuth M \& Sitte HH 2004 Oligomerization of the $\gamma$-aminobutyric acid transporter- 1 is driven by an interplay of polar and hydrophobic interactions in transmembrane helix II. Journal of Biological Chemistry 279 55728-55736. (doi:10.1074/ jbc.M409449200)

Lambert NA 2010 GPCR dimers fall apart. Science Signaling 3 pe12. (doi:10.1126/scisignal.3115pe12)

Law CJ, Maloney PC \& Wang DN 2008 Ins and outs of major facilitator superfamily antiporters. Annual Review of Microbiology 62 289-305. (doi:10.1146/annurev.micro.61.080706.093329)

Nyfeler B, Michnick SW \& Hauri HP 2005 Capturing protein interactions in the secretory pathway of living cells. PNAS 102 6350-6355. (doi:10.1073/pnas.0501976102)

Pascual JM, Wang D, Lecumberri B, Yang H, Mao X, Yang R \& De Vivo DC 2004 GLUT1 deficiency and other glucose transporter diseases. 
European Journal of Endocrinology 150 627-633. (doi:10.1530/eje. $0.1500627)$

Ridilla M, Narayanan A, Bolin JT \& Yernool DA 2012 Identification of the dimer interface of a bacterial $\mathrm{Ca}^{2+} / \mathrm{H}^{+}$antiporter. Biochemistry 51 9603-9611. (doi:10.1021/bi3012109)

Saier MH Jr, Tran CV \& Barabote RD 2006 TCDB: the Transporter Classification Database for membrane transport protein analyses and information. Nucleic Acids Research 34 D181-D186. (doi:10.1093/ nar/gkj001)

Schwartz CE, May MM, Carpenter NJ, Rogers RC, Martin J, Bialer MG, Ward J, Sanabria J, Marsa S, Lewis JA et al. 2005 Allan-Herndon-Dudley syndrome and the monocarboxylate transporter 8 (MCT8) gene. American Journal of Human Genetics 77 41-53. (doi:10.1086/431313)

Seidel S, Singer EA, Just H, Farhan H, Scholze P, Kudlacek O, Holy M, Koppatz K, Krivanek P, Freissmuth M et al. 2005 Amphetamines take two to tango: an oligomer-based counter-transport model of neurotransmitter transport explores the amphetamine action. Molecular Pharmacology 67 140-151. (doi:10.1124/mol.67.1.140)

Smith VE, Read ML, Turnell AS, Sharma N, Lewy GD, Fong JC, Seed RI, Kwan P, Ryan G, Mehanna H et al. 2012 PTTG-binding factor (PBF) is a novel regulator of the thyroid hormone transporter MCT8. Endocrinology 153 3526-3536. (doi:10.1210/en.2011-2030)

Sun L, Zeng X, Yan C, Sun X, Gong X, Rao Y \& Yan N 2012 Crystal structure of a bacterial homologue of glucose transporters GLUT1-4. Nature 490 361-366. (doi:10.1038/nature11524)

Teichmann A, Rutz C, Kreuchwig A, Krause G, Wiesner B \& Schulein R 2012 The pseudo signal peptide of the corticotropin-releasing factor receptor type $2 \mathrm{~A}$ prevents receptor oligomerization. Journal of Biological Chemistry 287 27265-27274. (doi:10.1074/jbc.M112.360594)

Visser WE, Friesema EC, Jansen J \& Visser TJ 2008 Thyroid hormone transport in and out of cells. Trends in Endocrinology and Metabolism 19 50-56. (doi:10.1016/j.tem.2007.11.003)

Visser WE, Philp NJ, van Dijk TB, Klootwijk W, Friesema EC, Jansen J, Beesley PW, Ianculescu AG \& Visser TJ 2009 Evidence for a homodimeric structure of human monocarboxylate transporter 8 . Endocrinology 150 5163-5170. (doi:10.1210/en.2009-0699)

Visser WE, Friesema EC \& Visser TJ 2011 Minireview: Thyroid hormone transporters: the knowns and the unknowns. Molecular Endocrinology 25 1-14. (doi:10.1210/me.2010-0095)

Visser WE, Vrijmoeth P, Visser FE, Arts WF, van Toor H \& Visser TJ 2013 Identification, functional analysis, prevalence and treatment of monocarboxylate transporter 8 (MCT8) mutations in a cohort of adult patients with mental retardation. Clinical Endocrinology 78 310-315. (doi:10.1111/cen.12023)

Watson RT \& Pessin JE 2001 Intracellular organization of insulin signaling and GLUT4 translocation. Recent Progress in Hormone Research $\mathbf{5 6}$ 175-193. (doi:10.1210/rp.56.1.175)

Xu Y, Tao Y, Cheung LS, Fan C, Chen LQ, Xu S, Perry K, Frommer WB \& Feng L 2014 Structures of bacterial homologues of SWEET transporters in two distinct conformations. Nature 515 448-452. (doi:10.1038/ nature13670)

Yin Y, He X, Szewczyk P, Nguyen T \& Chang G 2006 Structure of the multidrug transporter EmrD from Escherichia coli. Science 312 741-744. (doi:10.1126/science.1125629)

Received in final form 24 November 2014

Accepted 26 November 2014
() 2015 Society for Endocrinology Printed in Great Britain 